\title{
Mutation screening of brain-expressed $X$-chromosomal miRNA genes in 464 patients with nonsyndromic $X$-linked mental retardation
}

\author{
Wei Chen ${ }^{1,7}$, Lars R Jensen ${ }^{1,7}$, Jozef Gecz ${ }^{2}$, Jean-Pierre Fryns ${ }^{3}$, Claude Moraine ${ }^{4}$, \\ Arjan de Brouwer ${ }^{5}$, Jamel Chelly ${ }^{6}$, Bettina Moser ${ }^{1}$, H Hilger Ropers ${ }^{1}$ and Andreas W Kuss ${ }^{*}{ }^{1}$ \\ ${ }^{1}$ Max Planck Institute for Molecular Genetics, Berlin, Germany; ${ }^{2}$ Department of Genetic Medicine, Women's and \\ Children's Hospital, Adelaide, Australia; ${ }^{3}$ Centre for Human Genetics, University of Leuven, Leuven, Belgium; ${ }^{4}$ Service \\ de Génétique et INSERM U316, Hôpital Bretonneau, Tours, France; ${ }^{5}$ Department of Human Genetics, Radboud \\ University Medical Centre, Nijmegen, The Netherlands; ${ }^{6}$ INSERM 129-ICGM, Faculté de Médecine Cochin, Paris, \\ France
}

MiRNAs are small noncoding RNAs that control the expression of target genes at the post-transcriptional level and have been reported to modulate various biological processes. Their function as regulatory factors in gene expression renders them attractive candidates for harbouring genetic variants with subtle effects on IQ. In an attempt to investigate the potential role of miRNAs in the aetiology of X-linked mental retardation, we have examined all 13 known, brain-expressed X-chromosomal miRNAs in a cohort of 464 patients with non-syndromic X-linked MR and found four nucleotide changes in three different pre-miRNA hairpins. All the observed changes appear to be functionally neutral which, taken together with the rarity of detected nucleotide changes in miRNA genes, may reflect strong selection and thus underline the functional importance of miRNAs.

European Journal of Human Genetics (2007) 15, 375-378. doi:10.1038/sj.ejhg.5201758; published online 20 December 2006

Keywords: MiRNA; X-linked mental retardation; genetic modifier; mutation; brain

MiRNAs constitute an abundant class of small noncoding RNAs that regulate gene expression on the messenger level through sequence-specific base pairing. Recently, several miRNAs have been implicated in a variety of biological processes including developmental timing, programmed cell death, haematopoietic lineage differentiation, insulin secretion, and brain morphogenesis. ${ }^{1-5}$ It is therefore conceivable that defects in miRNAs play an important role in human genetic diseases.

*Correspondence: Dr AW Kuss, Max Planck Institute for Molecular Genetics, Ihnestrasse 73, 14195 Berlin, Germany.

Tel: + 4930 84131253; Fax: + 493084131383 ;

E-mail: kuss_a@molgen.mpg.de

${ }^{7}$ These authors contributed equally to this work.

Received 8 June 2006; revised 6 November 2006; accepted 8 November 2006; published online 20 December 2006
Mental retardation (MR) is a very heterogeneous disorder with a strong influence of hereditary factors. Males are significantly more often affected than females, which suggested an important role of X-linked genes. ${ }^{6}$ This has been borne out by the identification of causative mutations in $>20$ protein-coding genes for nonsyndromic, or 'pure', X-linked MR (XLMR), ${ }^{7}$ but recent epidemiological studies have shown that disease-causing mutations underlying X-linked recessive MR can explain at most half of the male excess observed. ${ }^{8-10}$ Mandel and Chelly ${ }^{11}$ have speculated that part of the male excess might be due to the existence of X-chromosomal modifiers with more subtle effects on the IQ. This model would also explain the finding that mutations in the ARX gene are significantly more common in large XLMR families with many affected males than in small families or sporadic male patients. Moreover, the 
existence of such modifiers would fit the observation that the male excess is highest in mild forms of MR. ${ }^{12}$ So far, however, there is no empirical evidence for the existence of sequence variants on the X-chromosome that act as modifiers of intelligence.

MiRNAs are interesting candidates for harbouring such variants because individual miRNAs can regulate the expression of several different target genes, whereas a single target gene may be under the control of several different miRNAs. ${ }^{13}$ Therefore, individual mutations in miRNAs may not be sufficient to cause disease but still able to act as genetic modifiers.

In order to investigate the involvement of miRNA mutations in XLMR, either as modifiers of intelligence or as sole causes of XLMR, we have examined all known, brain-expressed X-chromosomal miRNAs in a cohort of 464 mostly small families with nonsyndromic XLMR. This led to the identification of four nucleotide changes, located in three different miRNA genes.

\section{Materials and methods}

The sequences of pre-miRNA and the corresponding genome regions were obtained from the microRNA registry (http://www. sanger.ac.uk/software/Rfam/). Thirteen brain-expressed X-chromosomal miRNAs (see Table 1) were selected based on the expression data published by Bentwich et al. ${ }^{14}$ Genome regions covering the 13 premiRNAs were screened in a panel of 464 XLMR patients collected through the European XLMR consortium (http:// www.euromrx.com/). Of the 464 patients, 33 belong to families with defined linkage intervals on the $\mathrm{X}$ chromosome whereas the remaining 431 males are from small families with possible XLMR. For variants identified in patients, a panel of 183 male controls was screeened.

After PCR amplification (see Table 1 for primer sequences; reaction conditions are available upon request), patient PCR products were pairwise pooled for mutation screening by denaturing high-performance liquid chromatography (DHPLC with the WAVE ${ }^{\circledR}$, Transgenomic), using $2-4$ different temperatures as described previously. ${ }^{15}$ Mutations were verified by Sanger sequencing.

\section{Results and discussion}

MiRNAs are initially transcribed as long RNA precusor (pri-miRNAs) and then processed in the nucleus by the endonuclease Drosha to form approximately 60-nucleotide hairpin structures (pre-miRNAs). After export into the cytoplasm Dicer, a second endonuclease, catalyses the maturation of pre-miRNAs into approximately $22 \mathrm{nt}$ RNA duplexes. ${ }^{16}$ Based on the assumption that mutations, either in the mature miRNA and/or the hairpin pre-miRNA are most likely to affect the processing and/or target selection of miRNAs, the pre-miRNA sequences of all brain-expressed miRNAs were screened. Of the 13 screened pre-miRNAs, four nucleotide changes in three different pre-miRNA hairpins (Figure 1) were identified in four unrelated patients. Each of the four changes occurred only once in our panel of 464 patients. None of the changes is listed as a known SNP in the relevant databases, but one of them (the variation in mir223) was subsequently found in one out of 183 controls. The two variations found in mir-222 co-segregated with the disease in the respective families, whereas the change in mir-363 is absent in the proband's affected brother. All four changes are located outside the mature miRNA sequences.

The variations found in mir-363 and the $\mathrm{C}$ to $\mathrm{U}$ change in mir-222 are located in the stem regions of the respective pre-miRNA hairpins. Whereas the original sequences of these sites lead to $C$ to $G$ pairing, the two changes result in $\mathrm{U}$ to $\mathrm{G}$ pairing. Therefore, these variations do not alter the integrity of the stem and could be functionally neutral. Similarly, the A to $U$ change in mir-222 does not change the mismatch status of the position and thus may not affect the stem stability either. However, it has been reported that during miRNA maturation, Drosha cuts

Table 1 miRNA coordinates and PCR amplicons

\begin{tabular}{|c|c|c|c|c|c|}
\hline$I D$ & Start ${ }^{\mathrm{a}}$ & End $^{\mathrm{a}}$ & Forward primer & Reverse primer & Amplicon (bp) \\
\hline $\operatorname{mir}-222$ & 45362675 & 45362784 & CCAGAAGGCAAAGGATCACC & TGCCCAАTAАTСТСТСТCAGG & 166 \\
\hline $\operatorname{mir}-221$ & 45361839 & 45361948 & TTCTTGCGGTCCTITCTCTG & CССAGCATTTСТGAСTGTTG & 344 \\
\hline $\operatorname{mir}-362$ & 49476608 & 49476672 & AAACACACAAAAAGGGCAGG & TGAAAGAAGGGGACAGGTTG & 167 \\
\hline lef-7f-2 & 53467174 & 53467256 & TTCTCATGAAGTGCCACACC & AGGCTGAAGATGGACACTGG & 306 \\
\hline $\operatorname{mir}-223$ & 65021733 & 65021842 & TCССTATTCTGGTGCTTTGG & TGAGAACTTGGTGCTTGGTG & 327 \\
\hline $\operatorname{mir}-363$ & 133028928 & 133029002 & TGTAGCGCTGATCAGGAATG & TTCATGCGTATCTCCAGCAC & 304 \\
\hline mir-19b-2 & 133029221 & 133029316 & TGCTGGAGATACGCATGAAG & AACAGATGGTGGGGACTGTG & 315 \\
\hline mir-92-2 & 133029088 & 133029162 & TGTAGCGCTGATCAGGAATG & TTCATGCGTATCTCCAGCAC & 304 \\
\hline mir-106a & 133029748 & 133029828 & AGGGGCATTIAGGGCAGTAG & TGAGGACGGAAAAGAAGAGC & 286 \\
\hline mir-504 & 137475392 & 137475474 & ATTTTCATGAAGCATTGGGG & TACAGGGGACGAACTGATCC & 342 \\
\hline mir-105-1 & 151231259 & 151231339 & AACAAATGGCTITGGTCCAG & TGGATGTCAGCTTCTGTTGC & 155 \\
\hline mir-105-2 & 151233452 & 151233532 & GCTTTGTTCCAGAAGCAGATG & TGGTATCAGCTCATGTTGCAG & 148 \\
\hline mir-505 & 138731827 & 138731910 & ATGAGCAAATGAATGGGAGC & CTTGAAGCAGAAGAGCAGCC & 188 \\
\hline
\end{tabular}

${ }^{a}$ Chromosome position of miRNA in bp, based on the NCBI genome build 35 . 

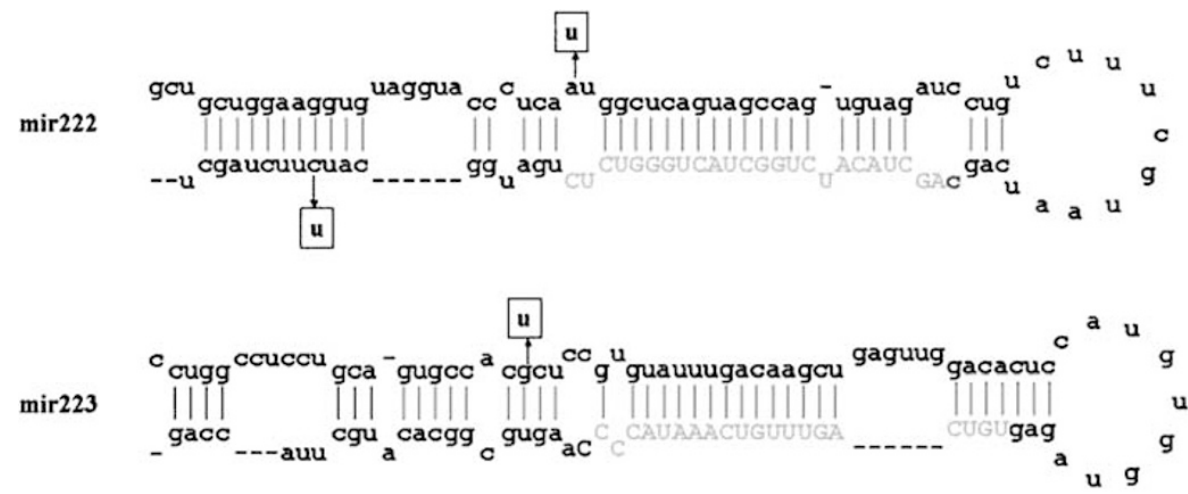

$\operatorname{mir}-363$

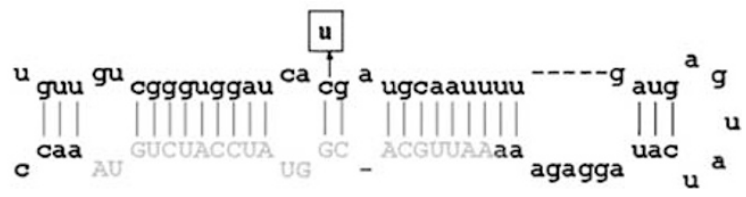

Figure 1 Variations in pre-miRNA sequences: pre-miRNA hairpins are shown, with gray capital letters indicating the respective mature sequence. Boxed letters represent the observed sequence changes.

pre-miRNAs at a distance of $\sim 22 \mathrm{nt}$ from the terminal loop-stem junction. ${ }^{17}$ If this is also the case for mir-222, the A to $U$ change would be quite close to the Drosha cleavage site and consequently, it might be able to influence the processing of its RNA product. However, when using ectopically expressed mir-222 constructs a detectable influence on Drosha processing could not be observed (David Bartel and Jinkuk Kim, unpublished data).

In the present study, mutation screening was confined to the pre-miRNA hairpins. It cannot be excluded, that mutations outside the pre-miRNA might also interfere with miRNA processing by affecting the pri-miRNA structure and variations in promoter regions may influence miRNA expression. The analysis of these regions, however, requires the clarification of pri-miRNA structure and unambiguous definition of miRNA promoters, of which little is known so far.

This study represents the first large-scale mutation screening of miRNAs in patients with mental retardation. The rarity of the nucleotide changes found in the investigated pre-miRNA hairpins and the fact that most of them appear to be functionally neutral may reflect strong selection acting on these regions and thus indicate the general importance of the miRNA system. Although so far, our results have failed to show an obvious role of miRNAs in the aetiology of mental retardation, our investigations do not rule out this possibility. Dysfunction of genes that are important for brain structure and function may also result from genetic variation of miRNA target sequences, for example, in $3^{\prime}$ untranslated regions. The analysis of such target sequences in large cohorts of families may shed more light on the role of miRNAs in the pathogenesis of nonsyndromic XLMR.

\section{Acknowledgements}

We are grateful to Dr Robledo and Dr Siniscalco for providing patient material of family MRX26, and to M Amende and B Lipkowitz for excellent technical support. The study was supported by the EURO$M R X$ project, part of the European 'Quality of Life and Management of Living Resources Programme' QLG3-CT-2002-01810, and by the Collaborative Research Program (SFB) 577 of the Deutsche Forschungsgemeinschaft.

\section{References}

1 Reinhart BJ, Slack FJ, Basson M et al: The 21-nucleotide let-7 RNA regulates developmental timing in Caenorhabditis elegans. Nature 2000; 403: $901-906$.

2 Brennecke J, Hipfner DR, Stark A, Russell RB, Cohen SM: Bantam encodes a developmentally regulated microRNA that controls cell proliferation and regulates the proapoptotic gene hid in Drosophila. Cell 2003; 113: 25-36.

3 Chen CZ, Li L, Lodish HF, Bartel DP: MicroRNAs modulate hematopoietic lineage differentiation. Science 2004; 303: 83-86.

4 Poy MN, Eliasson L, Krutzfeldt J et al: A pancreatic islet-specific microRNA regulates insulin secretion. Nature 2004; 432: 226-230.

5 Giraldez AJ, Cinalli RM, Glasner ME et al: MicroRNAs regulate brain morphogenesis in zebrafish. Science 2005; 308: 833-838.

6 Stevenson RE, Schwarte CE, Schroer RJ: X-linked Mental Retardation. Oxford: Oxford University Press, 2000.

7 Ropers HH, Hamel BC: X-linked mental retardation. Nat Rev Genet 2005; 6: 46-57.

8 Fishburn J, Turner G, Daniel A, Brookwell R: The diagnosis and frequency of X-linked conditions in a cohort of moderately retarded males with affected brothers. Am J Med Genet 1983; 14: 713-724.

9 Biancalana V, Beldjord C, Taillandier A et al: Five years of molecular diagnosis of Fragile X syndrome (1997-2001): a collaborative study reporting $95 \%$ of the activity in France. Am J Med Genet A 2004; 129: 218-224.

10 Gronskov K, Hjalgrim H, Nielsen IM, Brondum-Nielsen K: Screening of the ARX gene in 682 retarded males. Eur J Hum Genet 2004; 12: 701-705.

11 Mandel JL, Chelly J: Monogenic X-linked mental retardation: is it as frequent as currently estimated? The paradox of the ARX 
(Aristaless X) mutations. Eur J Hum Genet 2004; 12: 689-693.

12 Leonard $\mathrm{H}$, Wen $\mathrm{X}$ : The epidemiology of mental retardation: challenges and opportunities in the new millennium. Ment Retard Dev Disabil Res Rev 2002; 8: 117-134.

13 Bartel DP: MicroRNAs: genomics, biogenesis, mechanism, and function. Cell 2004; 116: 281-297.

14 Bentwich I, Avniel A, Karov Y et al: Identification of hundreds of conserved and nonconserved human microRNAs. Nat Genet 2005; 37: 766-770.
15 Jensen LR, Lenzner S, Moser B et al: X-linked mental retardation: a comprehensive molecular screen of 47 candidate genes from a $7.4 \mathrm{Mb}$ interval in Xp11. Eur J Hum Genet, advance online publication, 13 September 2006.

16 Lee Y, Jeon K, Lee JT, Kim S, Kim VN: MicroRNA maturation stepwise processing and subcellular localization. EMBO J 2002; 21: $4663-4670$.

17 Han J, Lee Y, Yeom KH et al: Molecular basis for the recognition of primary microRNAs by the Drosha-DGCR8 complex. Cell 2006; 125: 887-901. 